\title{
Baroreflex activation therapy lowers BP
}

Despite using multiple pharmacological therapies, many patients are unable to get their blood pressure (BP) down to target levels. The Rheos pivotal trial investigators have now reported that, although their study did not meet some of its primary end points, baroreflex activation therapy (BAT) can lower BP in patients with drug-resistant hypertension. Further improvement of this technology is currently underway.

BAT involves the application of electrical impulses to the carotid sinus, to modulate deranged sympathovagal balance in patients with drug-resistant hypertension. This stimulation of the carotid baroreceptors results in the brain receiving the message that BP is increased and, consequentially, signals to reduce BP are sent to the kidneys, heart, and vasculature.

In the Rheos pivotal trial, the BAT device (Rheos ${ }^{\circledR}$ system, CVRx, Inc., Minneapolis, MN, USA) was activated 1 month after implantation in the 181 patients who made up 'Group A'. The remaining patients ( $n=84$; 'Group B') had their BAT devices activated 7 months after implantation (that is, 6 months after those of Group A). In total, Group A and Group B received BAT for 12 and 6 months, respectively. After optimization of therapy, which was performed over the first 4 months of BAT, $75 \%$ of patients were programmed to a unilateral pathway.

The proportion of Group A patients who experienced $\geq 10 \mathrm{mmHg}$ reduction in systolic BP during the first 6 months of BAT (54\%) was not 'super-superior' (superiority margin $>20 \%$ ) to that of Group B (46\%) at the same time point (that is, when Group B not yet had their BAT initiated). The trial did not, therefore, meet the primary end point of 'acute efficacy'. Nevertheless, in the 97 responders in Group A at the 6-month time point, 88\% still showed $\geq 65 \%$ of their acute response at the 12-month time point, meaning that the trial did meet the primary end point of 'sustained efficacy'.

By the end of the study, $81 \%$ of all patients had experienced $\mathrm{a} \geq 10 \mathrm{mmHg}$ reduction in systolic BP, and both groups of patients had a mean reduction in systolic BP of $\sim 35 \mathrm{mmHg}$, compared with their BP prior to device implantation. Furthermore, more than $50 \%$ of all patients had systolic BP $\leq 140 \mathrm{mmHg}$.

Although the primary end points of 'BAT safety' and 'device safety' were met in the

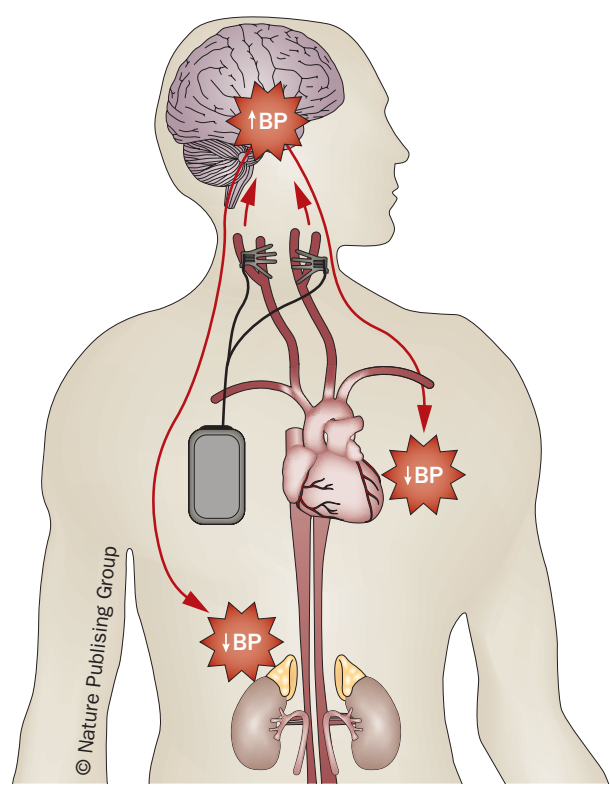

trial, that of 'procedural safety' was not. In total, 68 adverse events were associated with the procedure itself, including 25 transient or permanent nerve injuries.

Dr Suzanne Oparil, from the University of Alabama at Birmingham, USA, (who was not involved in the Rheos pivotal trial) is optimistic about the future of BAT for patients with drug-resistant hypertension, but highlights that "a shortcoming of the BAT device that was deployed in this study, and the procedures used to deploy it, is morbidity related to the extensive neck dissection required to place the device". Notably, though, Dr John Bisognano (lead author on the Rheos pivotal trial report) has told us that "the device is being improved and made smaller, and the surgical procedure [is being] improved and should be faster as well."

Dr Markus Schlaich from the Baker IDI Heart and Diabetes Institute, Australia, (who also was not a Rheos Pivotal trial investigator, but was an investigator in the Symplicity HTN-2 trial of catheterbased renal denervation in patients with drug-resistant hypertension) agrees with Dr Oparil and Dr Bisognano in that "it is worthwhile to further explore this approach". He suggests that it might be worth assessing only unilateral implantation in future BAT trials.

Bryony M. Mearns

\footnotetext{
Original article Bisognano, J. D. et al. Baroreflex activation therapy lowers blood pressure in patients with resistant hypertension: results from the double-blind, randomized, placebo-controlled Rheos pivotal trial. J. Am. Coll. Cardiol. 58, 765-773 (2011)
} 